\title{
Interview with Larry Guth
}

Toufik Mansour

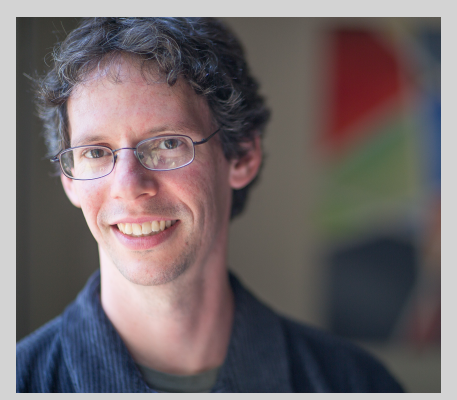

Larry Guth received a B.S. in mathematics from Yale University in 2000, and a Ph.D. in mathematics from the Massachusetts Institute of Technology (MIT) in 2005 under the supervision of Tomasz Mrowka. After getting his postdoctoral position at Stanford, he moved to the University of Toronto as an assistant professor. In 2011, Guth was appointed as professor of mathematics at the Courant Institute. Since 2012, he is a professor of mathematics at MIT, and in 2020 he was made a Claude Shannon professor. Guth's research interests are in metric geometry, harmonic analysis and extremal combinatorics. In 2010, Guth won an Alfred P. Sloan Fellowship. In 2013, the American Mathematical Society (AMS) awarded him the Salem Prize in mathematics, for outstanding contributions to analysis. In 2014, he received a Simons Investigator Award. In 2015, Guth received the Research Prize of the Clay Mathematics Institute and was awarded the New Horizons in Mathematics Prize "for ingenious and surprising solutions to long-standing open problems in symplectic geometry, Riemannian geometry, harmonic analysis, and combinatorial geometry." In 2020, Guth received the Bôcher Memorial Prize of the AMS and the Maryam Mirzakhani Prize in mathematics. He is a Fellow of the AMS.

Mansour: Professor Guth, first of all, we would like to thank you for accepting this interview. Would you tell us broadly what combinatorics is?

Guth: Combinatorics is about studying finite objects, like finite (abstract) sets, or a finite set of numbers or a finite set of circles in the plane. Questions from number theory, like questions about prime numbers or questions about integer solutions of equations, are finite in this sense, but partly for historical reasons, they are considered a separate field. In the 20th century, people realized that there are many other interesting questions about finite objects outside of number theory. These questions and the community of people working on them are combinatorics.

People in combinatorics have made a particular effort to seek out questions that are easy to state. There are many really difficult open problems in combinatorics which high school students can understand. In my opinion, this is an important part of the culture of the field.

Mansour: What do you think about the development of the relations between combinatorics and the rest of mathematics?

Guth: Personally, I did not start out studying combinatorics, and the only way I became involved was because of some relations between combinatorics and other parts of math. For instance, the Szemeredi-Trotter problem is a combinatorial problem about the intersection patterns of finite sets of lines in the plane, and the solution to the problem turns out to involve topology. This connection between combinatorics and topology is one of my favorite parts of combinatorics. The problem Szemeredi and Trotter solved is analogous to some problems in harmonic analysis. Tom Wolff discovered that analogy, and he learned ideas from the combinatorics community and applied them to study solutions of the wave

The authors: Released under the CC BY-ND license (International 4.0), Published: January 15, 2021

Toufik Mansour is a professor of mathematics at the University of Haifa, Israel. His email address is tmansour@univ.haifa.ac.il 
equation. Along the way, he introduced a circle of problems and ideas from combinatorial geometry to people working in harmonic analysis. I learned about these problems and ideas from Nets Katz. That had a big impact on my work. There are many other examples of connections between combinatorics and other parts of math.

Connections between one field and another are always interesting and important, but I think this may be especially true for combinatorics. On the one hand, combinatorics is distinguished by searching for questions that are very simple to pose. When those questions get connected to other branches of math and to more abstract structures, then I think it is a good sign for both the question and the abstract structure. It shows that the question leads us somewhere and it shows that the abstract structure connects to things.

Mansour: We would like to ask you about your formative years. What were your early experiences with mathematics? Did that happen under the influence of your family, or some other people?

Guth: I was incredibly fortunate with the mentors I had growing up. My father, Alan Guth, is a physicist and very interested in math, and we would talk a lot. He is a wonderful teacher. He taught me many things, and he was also happy to talk about questions he did not know the answers to. We would guess what the answer might be, and then we would try to prove it. If that did not work, we would try to disprove it. If that did not work, we would try to prove it again. Now I teach students myself, and it is very common that someone tries to prove something, and it does not work, and they get frustrated. Of course, it happens to me too. But sometimes, when we are in the right mood, we can say to ourselves, "That did not work. That is interesting. I wonder why it did not work..." I learned this mood when I was a kid talking math with my father.

Now I am a father. My children are four years old and two years old. When I watch them building a block tower or working on a jigsaw puzzle, I often think about doing math research and about the different moods that I go through when I am doing it. Sometimes, they are in a mood where they really want the tower to be tall. In that mood, they get frustrated when it falls down. But other times they build in a more curious mood, and when the tower falls down, they laugh and they start again. In the more curious mood, their hands are steadier and the towers are taller.

I also learned a lot from my father about writing and teaching. I remember, when we were talking, some times he would write down what we were talking about, and sometimes I would write down what we were talking about. When he wrote down what we were talking about, you saw the first thought and then the second thought and then the third thought. The pages were numbered and you could read it over later and see just what we thought. When I wrote down what we were talking about, the second thought was in the bottom right corner and the third thought was diagonally across the top left and the fourth thought was sideways along the right margin... It took me something like ten years to learn how to write thoughts down one at a time.

Mansour: Your father, Alan Guth, is an eminent theoretical physicist and cosmologist. Have you ever hesitated when choosing between mathematics and physics to pursue your research career?

Guth: I did not actually. We talked about physics too, but I remember our math conversations more vividly. Our conversations mostly followed what I was interested in talking about, and my interests went in a more mathematical direction.

Mansour: Were there specific problems that made you first interested in combinatorics?

Guth: When I was a postdoc, I learned about the Szemerdi-Trotter theorem ${ }^{1,2}$ from Matt Kahle, and I found it very interesting. I was interested in the Kakeya problem from harmonic analysis. Around that same time, Zeev Dvir ${ }^{3}$ proved the finite field Kakeya conjecture. The argument used ideas from errorcorrecting codes, and it was connected to combinatorics. I was very interested in that and I spent a long time trying to adapt those ideas to other problems. I met Nets Katz around

\footnotetext{
${ }^{1}$ E. Szemerédi and W.T. Trotter, Extremal problems in discrete geometry, Combinatorica 3(3-4) (1983), 381-392

${ }^{2}$ E. Szemerédi and W.T. Trotter, A combinatorial distinction between the Euclidean and projective planes, European J. Combin. 4(4) (1983), 385-394.

${ }^{3}$ Z. Dvir, On the size of Kakeya sets in finite fields, J. Amer. Math. Soc. 22 (2009), 1093-1097.
} 
that time, and he introduced me to a bunch of problems in combinatorial geometry that have some analogy or connection to the Kakeya problem. These problems included the joints problem, the unit distance problem, the distinct distance problem, and analogues of the Szemeredi-Trotter theorem using other kinds of curves in place of lines. Those are the problems that got me into combinatorics, and I still think they are very interesting.

Mansour: What was the reason you chose MIT for your Ph.D. and your advisor, Tomasz Mrowka?

Guth: I liked the atmosphere among the students at MIT, and I also liked being in Boston and close to my family. Coming out of undergrad, I was most interested in Fourier analysis, which I had learned about from Peter Jones and Ronald Coifman, but I really was not sure what I wanted to work on. MIT was not actually the best place for Fourier analysis, but there was clearly a lot of good math and it felt like a good place for me.

When I was a first-year Ph.D. student, I was not really able to understand what any of the professors were working on. Tom had a group of six or seven students. I knew some of them and I liked the community of this group. We had a weekly seminar meeting where we took turns reading papers and presenting them to each other, and that was a great experience for me. I had never read a math paper as an undergrad, or as a first year grad student. Learning to read a paper and digest it and present it was challenging and exciting. My first presentation had about one mistake every ten minutes, and Tom pointed them out with gentle questions. I had not learned how much work it took me to digest a piece of math and understand it. After that, I wrote about eight pages of notes for each presentation, and I wrote more than one draft of the trickiest parts.

Mansour: What was the problem you worked on in your thesis?

Guth: In Gromov and Lawson's work on scalar curvature ${ }^{4}$, they bring up mappings between $n$-dimensional Riemannian manifolds that decrease all the 2-dimensional areas. I got very interested in these mappings and I wrote my thesis about them.

Mansour: What would guide you in your research? A general theoretical question or a specific problem?

Guth: In that thesis research, I was guided by analogies. There is a lot known about mappings that decrease all 1-dimensional lengths. I would work through those ideas and try to adapt them to mappings that decrease all the 2-dimensional areas. For example, there is a fundamental theorem in the area called the Lipschitz extension theorem, which says that if $S \subset \mathrm{R}^{n}$ is any set, and $f: S \rightarrow \mathrm{R}$ is a distance-decreasing map, then $f$ extends to a length-decreasing map from $\mathrm{R}^{n}$ to $\mathrm{R}$. I wanted to know if there was some analogue of this theorem involving 2-dimensional areas instead of 1-dimensional lengths.

I worked on that for a while, but did not make any progress, and got frustrated. Eventually, I decided to give up and just work out some simple examples of area-decreasing maps. It seemed natural to me that among all the maps from one $n$-dimensional box to another $n$-dimensional box, the linear map would have the best area-decreasing properties. I did not think it would be that hard or that interesting, but I figured I would work on that and at least I would prove something. So I gave up on my "real" problem, and I tried to prove that little conjecture about rectangular boxes. That little conjecture turned out to be false! My thesis problem was figuring out which map has the best area-decreasing properties. (I was able to do this in some dimensions - the general problem is still open.)

The moment I figured out that my little conjecture was false was an important moment for me becoming a math researcher. The analogies I had started with were not all that helpful. Once I discovered that my intuition about this little conjecture was wrong, there were a lot of followup questions and I had a lot of concrete things to do that helped me understand the area better.

Sometimes in research, I am guided by something that bothers me. I once went to a panel discussion about mentoring graduate students, and Ingrid Daubechies said that she teachers her students to "be ornery read-

\footnotetext{
${ }^{4}$ M. Gromov and H.B. Lawson, Jr., Spin and scalar curvature in the presence of a fundamental group. I, Ann. of Math. 111:2 (1980), 209-230.
} 
ers". In other words, she encourages them to read papers and pay attention to what bothers them. Recently, I have been working a lot on decoupling theory in Fourier analysis, following the work of Bourgain and Demeter. Their proof of the decoupling conjecture for the paraboloid ${ }^{5}$ came as a complete shock to me, and I have been studying it and working in the area ever since. A key idea in the proofs is to combine information from different scales. There are a bunch of different tricks for doing this. The proof of decoupling involves four distinct tricks for combining information from different scales. And in applications, the decoupling theorem itself is used at different scales. Combining information from many different scales seems natural and important to me. But having five different tricks for doing so does not feel natural - it bothers me. I feel like we have not yet found the clearest way of understanding it. I have been thinking about that for the last several years.

Mansour: When you are working on a problem, do you feel that something is true even before you have the proof?

Guth: Yes, I do. And sometimes I am wrong.

Mansour: What three results do you consider the most influential in combinatorics during the last thirty years?

Guth: I do not feel qualified to answer this question. I only know a very narrow part of combinatorics. But I will mention a few things that I find exciting and that I would like to learn. I would like to learn about Gowers's work ${ }^{6,7}$ on arithmetic progressions using higher-order Fourier analysis. I would like to learn about the Green-Tao theoremm ${ }^{8}$. The Green-Tao theorem is related to a lot of things, but one, in particular, I would like to learn about is the analogue of Szemeredi's regularity lemma ${ }^{9}$ in the context of sparse graphs. I am not sure if this counts as combinatorics exactly, but I would like to learn more about the PCP theorem and hardness of approximation.

Mansour: What are the top three open ques- tions in your list?

Guth: The problems I have spent the most time wondering about are the Kakeya problem ${ }^{10,11}$ and its cousins. In combinatorics, one of my favorite questions is the inverse problem for the Szemeredi-Trotter theorem. I recently learned about an open problem in additive combinatorics about the size of the sum set of a convex set. If $f(x)$ is a convex function, then the sequence of numbers $f(1), f(2), \ldots, f(n)$ is a convex set of size $n$. If $A$ is a convex set of size $n$, how big does the sum set $A+A$ need to be? There was some striking fairly recent work by Schoen and Shkredov ${ }^{12}$, but we are far from fully understanding.

Mansour: What do you think about the distinction between pure and applied mathematics that some people focus on? Is it meaningful at all in your own case? How do you see the relationship between so-called "pure" and "applied" mathematics?

Guth: So far I have only done pure math. I would really like to learn more applied math, but it is hard to find time for everything...

Mansour: Would you tell us about your interests besides mathematics?

Guth: When I was younger I was interested in acting and in theater. When I was in college, I spent a semester studying acting in Moscow and I did another workshop the summer after my first year of graduate school. My family used to go to the theater when I was growing up and there were many plays around our house that I used to read. For me, it was also a way of exploring different life experiences and trying out different ways of connecting with people. Once in a while I still have friends over and read a play out loud.

I started taking some tai chi classes in graduate school. I got interested in it partly through theater and partly because I used to have a little knee pain and back pain, and I think it helped with that. I really like the meditative side of it, and I still like to do a little bit when I get into the office in the morning.

\footnotetext{
${ }^{5}$ J. Bourgain and C. Demeter, The proof of the $l^{2}$ Decoupling Conjecture, Ann. of Math. 182 (2015), 351-389.

${ }^{6}$ T. Gowers, A new proof of Szemerédi's theorem, Geom. Funct. Anal. 11(3) (2001), 465-588.

${ }^{7}$ T. Gowers, Hypergraph regularity and the multidimensional Szemerédi theorem, Ann. of Math. 166(3) (2007), 897-946.

${ }^{8} \mathrm{~B}$. Green and T. Tao, The primes contain arbitrarily long arithmetic progressions, Ann. of Math. (2) 167 (2008), $481-547$.

${ }^{9}$ B. Green, T. Tao and T. Ziegler, An inverse theorem for the Gowers $U^{s+1}[N]-n o r m$, Ann. of Math. 176 (2012), $1231-1372$.

${ }^{10}$ A.S. Besicovitch, On Kakeya's problem and a similar one, Math. Z. 27:1 (1928), 312-320.

${ }^{11} \mathrm{~T}$. Tao, From Rotating Needles to Stability of Waves: Emerging Connections between Combinatorics, Analysis and PDE, Notices of the AMS. 48(3) (2001), 297-303.

${ }^{12}$ T. Schoen and I.D. Shkredov, On sumsets of convex sets, Combin. Probab. Comput. 20:5 (2011), 793-798.
} 
Mansour: Before we close this interview, we would like to ask some more specific mathematical questions. You have worked on problems from different fields of mathematics throughout your career. In your survey paper "Unexpected applications of polynomials in combinatorics," you write "I am a big admirer of hard problems that are simple to state. The most exciting - in my opinion - is a simply stated problem that is hard for a new reason." Would you elaborate more about this and give some examples of such problems? Are you working on a problem recently which is hard for a new reason?

Guth: There are many hard questions about primes that are simple to state, such as the twin primes conjecture. I think that is a very striking fact. In hindsight, because prime numbers are defined by crossing out numbers with factors and looking at what is left, it is hard to get a handle on what happens when we add prime numbers etc. Once one knows about this, it is not so hard to produce other simple questions about primes that are also hard to answer. Even though the definition of a prime number is simple, testing whether a number is prime is quite complicated, and so in hindsight, perhaps it is not so surprising that it is hard to know how often $n$ and $n+2$ are both prime.

Erdős posed the unit distance problem ${ }^{13,14}$ in the 1940s, and it remains wide open and looks hard. The reason it is hard is not related to the reason the twin primes conjecture is hard. As far as I know, there was not a previous problem that was hard for a similar reason. In fact, I have some trouble articulating why I believe the problem is hard. Actually I think a hard problem is the most interesting in this situation - when it is not so clear why it is hard. Then our research has two opposite goals: either to make progress on the problem or to explain why it is hard to make progress.

The unit distance problem asks for the maximum possible number of unit distances among $n$ points in the plane. Since we have $2 n$ degrees of freedom, it is not hard to get about $2 n$ unit distances. Examples with more than that many unit distances are rare, and they all have some special lattice structure. Those ex- amples only have slightly more unit distances, still less than $n^{1+\epsilon}$ asymptotically. Valtr ${ }^{15}$ discovered that the unit distance conjecture is false for other smooth convex norms. The examples still have lattice structure! So perhaps there is some general principle that examples with many unit distances must have some kind of lattice structure. That would be very striking - and reminiscent of several other problems in combinatorics and Fourier analysis where the known examples have a lot of lattice structure, such as examples for the Szemeredi-Trotter theorem and examples for the Bourgain-Demeter decoupling theorem. If a set has a lot of unit distances, what kind of structure does it need to have, and how do we know it is there?

Mansour: Between 2006-2013, several very difficult combinatorial problems have been solved in an unexpected way using high degree polynomials. For instance, the finite field Nikodym and Kakeya problems, and Erdös distinct distance problem. You have already written a wonderful survey paper and a book on the topic called "Polynomial methods in Combinatorics." Would you tell us about the main ideas behind the polynomial method? Why is it so powerful?

Guth: In these problems and related problems, the extreme examples have polynomial structure. For example, a set of points is contained inside of a surface defined by (fairly low degree) polynomials. The polynomial method is a set of tools that helps figure out when extreme examples for different types of problems need to have this kind of polynomial structure. Mansour: You, with Nets Katz, introduced a variation of the polynomial method, often called polynomial partitioning, in your solution to the Erdös distinct distances problem in the plane. Would you tell us about the problem, polynomial partitioning method, and the higher dimensional cases?

Guth: For example, suppose we have a set of points $P$ in 3-dimensional space and a set of lines $L$ in 3 -dimensional space, and we want to estimate the number of incidences of $P$ and $L$ (the number of pairs $p \in P$ and $\ell \in L$ with $p \in \ell$ ). The worst case occurs when all the points and lines lie in a plane. Nets and I

\footnotetext{
${ }^{13}$ P. Brass, W.O. Moser and J. Pach, Research problems in discrete geometry, Springer, 2005.

${ }^{14}$ P. Erdös, On sets of distances of $n$ points, Amer. Math. Monthly 53 (1946), 248-250.

${ }^{15}$ P. Valtr, Strictly convex norms allowing many unit distances and related touching questions, preprint, 2005.
} 
proved that if not too many points or lines lie in a single plane, then there are significantly better estimates. It turns out that to analyze this problem, one should first check that the worst case occurs when most of the points and lines lie in a low degree algebraic surface. Then one analyzes the different surfaces, and one finds that only planes and degree 2 surfaces matter. Since only planes and degree 2 surfaces matter, why introduce all the higher degree surfaces in the proof? I think the reason is that there is a more robust phenomenon - for a broad range of problems in this spirit, the worst case occurs when most of the objects involved lie in an algebraic variety of fairly low degree. It so happens that for points and lines in 3-space, only planes and sometimes degree 2 surfaces matter, but this last point is a little bit of a coincidence. The more general robust phenomenon does involve higher degree varieties, and the more general and robust phenomena are often easier to prove.

This estimate about lines in 3-space was the main step in realizing an approach to the distinct distance problem that was invented by Sharir and Elekes ${ }^{16}$.

There are many higher dimensional versions of these problems. One can ask about incidences between lines in $n$-space and more generally between low degree varieties in $n$-space. The higher dimensional distinct distance problem leads to incidence problems of this kind. Most of these higher dimensional versions are open, although Miguel Walsh recently made a lot of progress on them.

Mansour: Is there a connection between Noga Alon's combinatorial Nullstellensatz and the polynomial method?

Guth: They have a somewhat similar flavor because they both involve doing linear algebra on the space of polynomials. There are a number of other cool theorems and proofs in combinatorics that also involve linear algebra on the space of polynomials - one good source for them is the book 'Linear algebra methods in combinatorics' by Babai and Frankl. The recent breakthrough in the cap set problem ${ }^{17}$ by Croot, Lev, and $\mathrm{Pach}^{18}$ is another example. Mansour: Would you list a few open questions from combinatorics such that in their solutions you expect that polynomial method will play an important role?

Guth: For several years, people used to ask me if this or that problem might be related to the polynomial method. I was not very good at guessing the answer - and the answer was usually no. Unless the problem was very similar to a problem that had been solved using the polynomial method, I had no special insight. I would tell people that a problem was a good candidate if the worst known example had some polynomial structure.

In the last ten years, two of the most exciting applications of the polynomial method were the work on finite field sum-product estimates using Rudnev's ${ }^{19}$ point-plane incidence bound and the work of the cap set problem by Croot, Lev, and Pach. In these cases, there is not any interesting example with polynomial structure, so my criterion would not have guessed that the polynomial method should be useful!

Mansour: Terence Tao has the following comment on the polynomial method: "What I would like to see more of in the future is more development of the somewhat vague idea of the "Zariski complexity" of various sets, by which I mean something like the least degree of a nontrivial polynomial which vanishes on that set. One can view the polynomial method as the strategy of comparing upper and lower bounds on the Zariski complexity of sets to obtain nontrivial combinatorial consequences. I have the vague feeling that ultimately, such notions of complexity should play as prominent a role in these sorts of combinatorial problems as existing notions of "size" for such sets, such as cardinality, dimension, or Fourier uniformity." 20 Would you elaborate on this comment to make it more accessible to a researcher who is new to the field? What are your thoughts in this direction?

Guth: Suppose $\mathrm{F}$ is a field. If $X \subset \mathrm{F}^{n}$ is a set, we can define $\operatorname{Deg}(X)$ as the smallest

\footnotetext{
${ }^{16} \mathrm{G}$. Elekes and M. Sharir, Incidences in three dimensions and distinct distances in the plane, Combin. Probab. Comput. 20:4 (2011), 571-608

${ }^{17}$ J.A. Grochow, New applications of the polynomial method: The cap set conjecture and beyond Bull. AMS 56:1 (2019), 29-64.

${ }^{18}$ E. Croot, V.F. Lev and P.P. Pach, Progression-free sets in $\mathbb{Z}_{4}^{n}$ are exponentially small, Ann. of Math. (2) 185:1 (2017), 331-337.

${ }^{19} \mathrm{M}$. Rudnev, On the number of incidences between points and planes in three dimensions, Combinatorica 38 (2018), 219-254.

${ }^{20}$ See, https://mathoverflow.net/a/43549
} 
degree of a non-zero polynomial that vanishes on $X$. This definition plays a big role in the polynomial method, especially in some of the early work. For a random set $X \subset \mathrm{F}^{n}, \operatorname{Deg}(X)$ is approximately $|X|^{1 / n}$. If $\operatorname{Deg}(X)$ is much smaller than this, then we can say informally that $X$ has some algebraic structure. Many proofs in the polynomial method begin by supposing that $X$ has some interesting combinatorial geometric property, and then showing that $\operatorname{Deg}(X)$ is small. Once this happens, we know that $X$ has some algebraic structure, and we can often learn more about $X$ by applying tools from algebraic geometry.

So $\operatorname{Deg}(X)$ is an important player in the polynomial method. But it is not the final word, especially when $n$ is large. If $X$ is contained in an $(n-1)$-plane in $n$-space, then $\operatorname{Deg}(X)=1$. But a set contained in a 9-plane can still be pretty complicated. If a set is contained in a 2-plane, should not it be simpler than a set that is just contained in a 9-plane? They both have $\operatorname{Deg}(X)=1$. So we should refine $\operatorname{Deg}(X)$ by taking into account not just algebraic hypersurfaces but algebraic varieties of all dimensions. This is done in a somewhat ad hoc way in various papers. Walsh's ${ }^{21}$ recent work is a big step towards studying varieties of all dimensions in a systematic way.

Mansour: You are the first winner of the Maryam Mirzakhani Prize in mathematics for mid-career mathematicians given by the National Academy of Sciences. The citation states that the award is for "developing surprising, original, and deep connections between geometry, analysis, topology, and combinatorics, which have led to the solution of, or major advances on, many outstanding problems in these fields." What do you think about the importance of being competent in different branches of mathematics? Do you have a concrete example in your research career where you made a significant progress on a research problem by using ideas or techniques from a completely different field?

Guth: On the one hand, dividing math into fields is somewhat artificial, and there are many relations between "different" fields. So knowing different parts of math is certainly helpful. On the other hand, learning things well takes time, and none of us has time for everything.

The idea of polynomial partitioning that we mentioned above was partly suggested by work of Gromov in metric geometry, a rather different part of math from combinatorics or from harmonic analysis. If you take two open sets in the plane, then there is always a line that bisects both sets. You can kind of convince yourself of that by imagining a line continuously rotating and shifting. At each angle, there is a shift that bisects the first set, and as we change the angle, the line sweeps across the second set until it bisects it as well. This idea is a special case of the ham sandwich theorem. Following Gromov, I was using variations on the ham sandwich theorem to study problems in metric geometry. Then the idea played a role in my work on harmonic analysis and combinatorics, especially in the work with Nets on polynomial partitioning.

Mansour: Your initial work was on the systolic geometry. Is there a connection between combinatorics and systolic geometry?

Guth: Well, I was very excited by the application of ham sandwich theorems in combinatorics. I learned the ham sandwich theorem in the context of metric geometry (a broader area that includes systolic geometry). After that, I looked for more connections between the fields. I personally did not find any other connection.

Gromov found another interesting connection between metric geometry and combinatorics: he found an analogy between "point-selection" theorems in combinatorics by Barany and others and the waist theorem in Riemannian geometry proven by Almgren and Gromov.

Mansour: Professor Larry Guth, I would like to thank you for this very interesting interview on behalf of the journal Enumerative Combinatorics and Applications.

${ }^{21}$ M.N. Walsh, The polynomial method over varieties, Invent. Math. 222 (2020), 469-512. 